\title{
Robust Ultrasound Probe Tracking: \\ Initial Clinical Experiences During \\ Robot-Assisted Partial Nephrectomy
}

\author{
Philip Pratt ${ }^{1}$, Alexander Jaeger ${ }^{2}$, Archie Hughes-Hallett ${ }^{3}$, Erik Mayer ${ }^{3}$, \\ Justin Vale ${ }^{3}$, Ara Darzi ${ }^{1,3}$, Terry Peters ${ }^{4}$, Guang-Zhong Yang ${ }^{1}$ \\ ${ }^{1}$ Hamlyn Centre for Robotic Surgery, Imperial College London, UK \\ ${ }^{2}$ Electrical and Electronic Engineering, University College Cork, Ireland \\ ${ }^{3}$ Department of Surgery and Cancer, Imperial College London, UK \\ ${ }^{4}$ Robarts Research Institute, University of Western Ontario, Canada \\ p.pratt@imperial.ac.uk
}

\begin{abstract}
PURPOSE: In order to assist in the identification of renal vasculature and tumour boundaries in robot-assisted partial nephrectomy, robust ultrasound probe calibration and tracking methods are introduced. Contemporaneous image guidance during these crucial stages of the procedure should ultimately lead to improved safety and quality of outcome for the patient, through reduced positive margin rates, segmental clamping, shorter ischaemic times and nephron-sparing resection.

METHODS: Small KeyDot markers with circular dot patterns are attached to a miniature pick-up ultrasound probe. Generic probe calibration is superseded by a more robust scheme based on a sequence of physical transducer measurements. Motion prediction combined with a reduced region-of-interest in the endoscopic video feed facilitate real-time tracking and registration performance at full $\mathrm{HD}$ resolutions.
\end{abstract}

RESULTS: Quantitative analysis confirms that circular dot patterns result in an improved translational and rotational working envelope, in comparison to the previous chessboard pattern implementation. Furthermore, increased robustness is observed with respect to prevailing illumination levels, and out-of-focus images due to relatively small endoscopic depths of field.

CONCLUSION: Circular dot patterns should be employed in this context as they result in improved performance and robustness. This facilitates clinical usage and interpretation of the combined video and ultrasound overlay. The efficacy of the overall system is demonstrated in the first human clinical case.

\section{Keywords}

ultrasound, tracking, image guidance, partial nephrectomy, robot-assisted 


\section{Purpose}

The image-enhanced approach to surgical intervention aims to improve patient safety and oncological outcome, and this is demonstrated particularly well in robot-assisted partial nephrectomy [1], where an accurate appreciation of the typically variable renal vasculature and tumour interface is of paramount importance. The provision of an image guidance system based on preoperative imaging alone goes some way to facilitating this goal [2], in the sense that the topological relationships between structures are relatively straightforward to illustrate macroscopically. But to reach full efficacy, such a system must be complemented by a contemporaneous imaging modality [3], immune to the undesirable effects of tissue deformation, and capable of resolving structure at a more detailed level. Ultimately, this should lead to reduced positive margin rates, the option of segmental clamping, shorter ischaemic times and nephron-sparing resection.

To this end, the use of intracorporeal 'pick-up' ultrasound transducers [4,5,6] is becoming prevalent, where the resulting 2D scan images are automatically registered and presented in real time within the 3D surgical scene. Recent studies $[4,7]$ have considered conventional tracking methods (e.g. electromagnetic or optical tracking of the distal probe), but the ubiquity of ferromagnetic materials and awkward lines of sight in the operating theatre present problems that invariably hinder translation of this technology into everyday clinical practice. The endoscope itself can double as a tracking device, thereby eliminating a number of intermediate coordinate systems and, therefore, sources of registration error. Visual identification of a geometric pattern rigidly attached to the probe is sufficient to estimate its pose, ultimately leading to direct registration $[8,9]$ of the ultrasound image.

Even so, the problem of how to create an accurate ultrasound probe tracking algorithm of this nature, sufficiently fast and robust to work in a live and unforgiving clinical environment, has yet to be solved adequately. These criteria are prerequisites for widespread adoption of the technology. Therefore, learning from clinical experience, this study extends previous work [10] by adopting the principle of simplicity, and introducing a tracking pattern made up of circular dots. It also considers the output of the system, where the psychophysical effects 
of the method chosen to display the registered and fused images play a crucial role in anatomical interpretation and ease of use of the surgical guidance platform.

\section{Methods}

\section{Circular dot tracking}

The marker used for tracking was an asymmetric circle KeyDot ${ }^{\circledR}$ pattern (Key Surgical Inc., Eden Prairie, MN, USA). The marker was initially attached to a 'dummy' probe for the purpose of testing. Figure 1 illustrates the design of the pattern comprising an asymmetric grid of circles with 3 rows and 7 columns. Vertical and horizontal dot spacing was measured using Vernier calipers at $1.19 \mathrm{~mm}$, with each dot having a diameter of $500 \mu \mathrm{m}$. This pattern design was chosen as dots are geometrically simpler than squares, and it was anticipated that circular structures are less affected by degrading image effects such as blooming and smudging (figure 1, right). The tracking algorithm for detecting circles does not rely on the intersection of edges, but instead relies on an efficient 'blob detector' approach. Intuitively, tracking dots should be more robust when the reliance on well-defined edge crossings is eliminated.
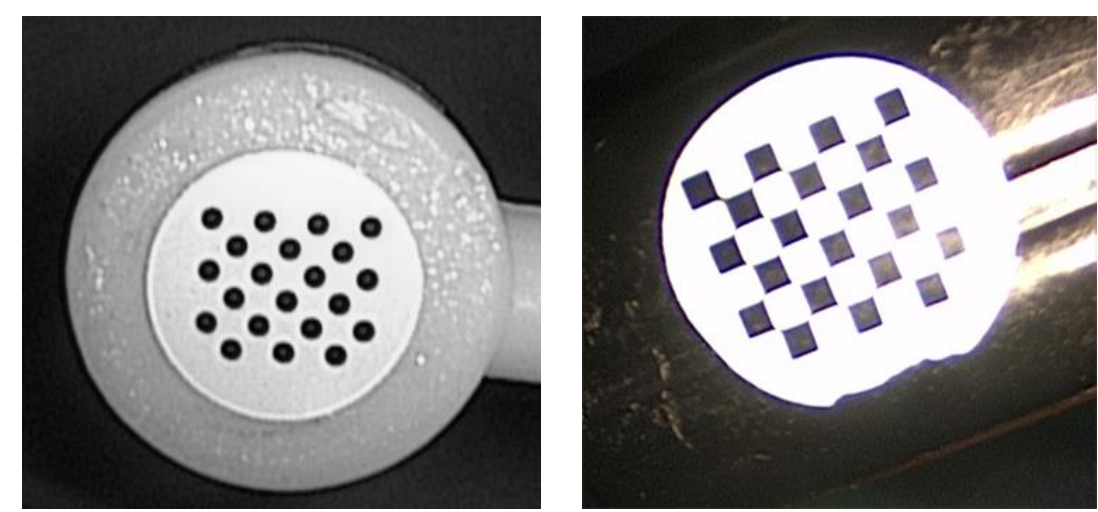

Figure 1. Asymmetrical circular dots pattern (left) and original chessboard pattern (right).

To facilitate repeatability, the OpenCV implementation of the 'simple blob detector' was employed [11]. At each invocation of findCirclesGrid(), the following steps are executed sequentially: 
- The source colour image is converted to a sequence of binary images by applying greyscale thresholds at equally spaced levels between specified minimum and maximum bounds.

- Connected components are extracted from each binary image using a contour extraction routine [12] utilising border following. The centres are found by computing the moments of each rasterised image.

- Blob centres are grouped by their coordinates across the sequence of thresholded images, in accordance with a specified 'minimum distance between blobs' parameter. Sufficiently large groups correspond to positively identified blobs.

- The final centre positions are estimated by a weighted mean. The use of an asymmetric pattern ensures that the sorted results are returned in a consistent manner.

The relevant detector parameters were set as follows: minThreshold $=10$; maxThreshold $=220 ;$ minDistBetweenBlobs $=5$ pixels; filterByArea $=$ TRUE; minArea $=25$ pixels $^{2} ;$ maxArea $=5000$ pixels $^{2} ;$ filterByIntertia $=$ TRUE; minInertiaRatio $=0.1 ;$ filterByConvexity $=$ TRUE; minConvexity $=0.95$; filterByColor $=$ TRUE; blobColor $=0 ;$ and minRepeatability $=2$.

\section{Tracking regions of interest}

The OpenCV findCirclesGrid() function is not accelerated by any means, in the sense that the internal components of the library function do not make use of parallel processing constructs nor heterogeneous computing APIs. This results in prohibitively long processing delays between frames when high-definition (HD) video streams are prevalent. A continuously updating cropping technique was developed to reduce the number of pixels that the function was required to process. On detecting the circular dot pattern initially, a set of rectangular coordinates whose area is proportional to the size of the pattern are calculated such that the coordinates envelop the location of the pattern in the image plane (figure 2). This set of coordinates is used to crop the next video frame prior to calling findCirclesGrid(). The cropping process is repeated for every frame. The method significantly reduces the number of pixels required for processing as it removes the majority of redundant information from the image. The relative size of the cropping area can be adjusted using the scaling factor S. A higher scaling 
factor yields a larger cropping area, at the expense of a degradation in performance. Chosen empirically, a scaling factor of $\mathrm{S}=0.4$ was used during the experiments herein.

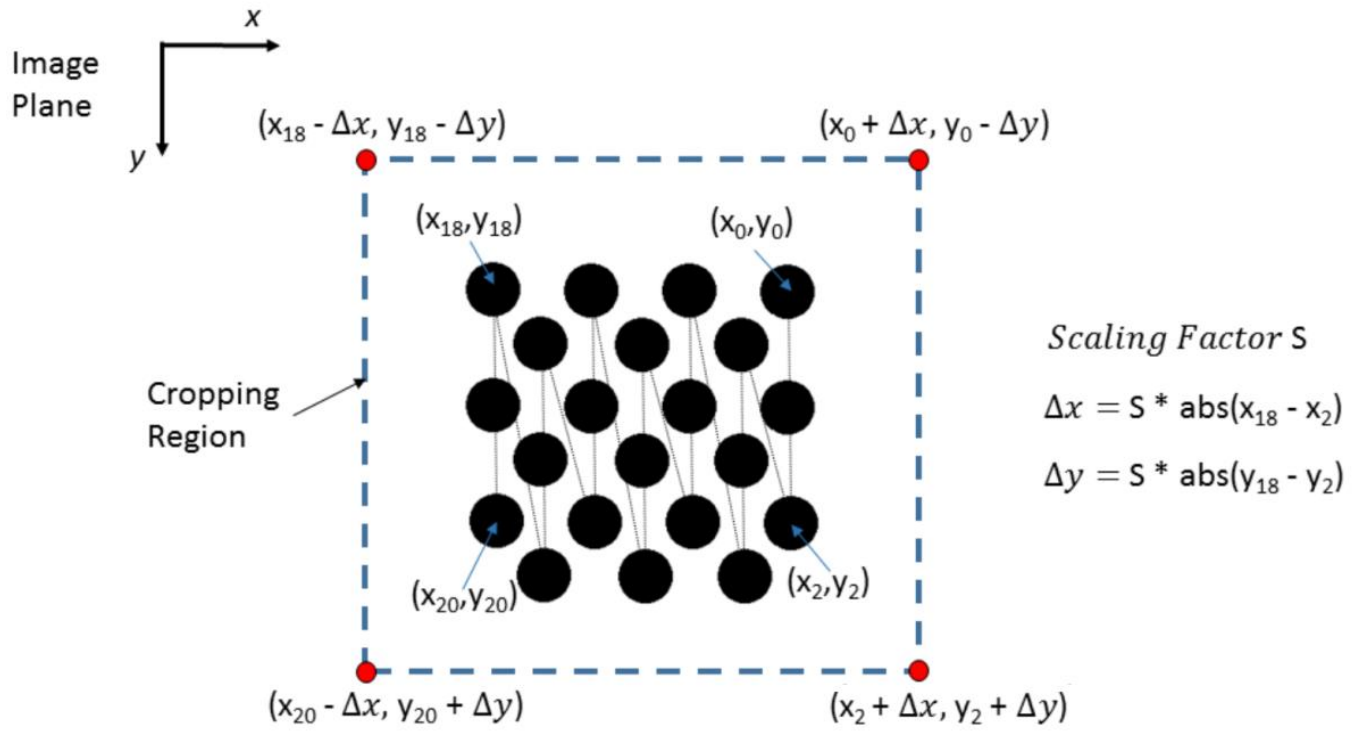

Figure 2. Calculating the appropriate cropping area around the circular dot pattern.

However, generation of a cropping region for use in future video frames inevitably causes failures when the pattern moves sufficiently far away. Since the cropping region calculation did not initially take into account the current velocity of the pattern, resulting in partial patterns and failures in recovering the location of all circular dots in the pattern, an additional step was included to account for such motion. Figure 3 illustrates the salient features of the calculation. The velocity of the pattern is estimated by taking the vector difference of the positions of the two previous consecutive cropping rectangles. The resulting displacement vector is multiplied by a scaling factor $\mathrm{S}_{\mathrm{v}}$ (set empirically to 0.5 ) and accumulated with the following cropping rectangle, thus amending the calculation with an approximate velocity-based 'prediction' of the location of the dot pattern in the next frame. Should the circular dot detection fail at any point, e.g. on account of a rapid motion, the region of interest is reset, a threshold step size of 30 is adopted and the entire image is processed. Otherwise the default size 10 is employed, allowing for finer steps during blob detection, permissible in the knowledge that the image has been cropped. 


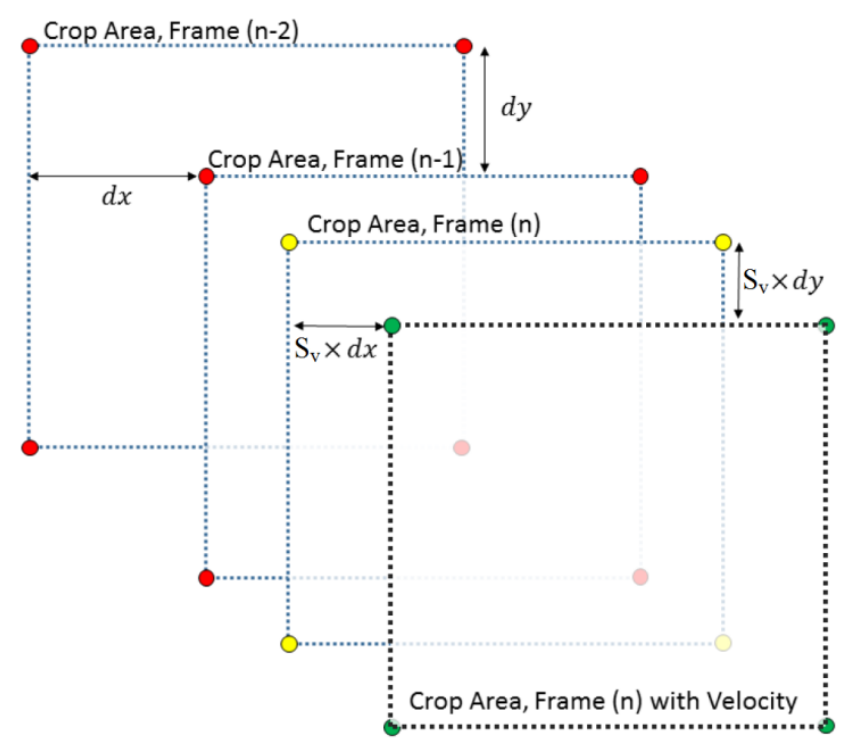

Figure 3. Cropping rectangles with velocity estimation and weighted accumulation.

\section{Ultrasound overlay}

Once the probe pattern has been recovered, its pose relative to the camera coordinate system can be estimated using the camera's calibrated [13] intrinsic properties. This transformation is concatenated with the constant image-to-pattern relationship (i.e. probe calibration) in order to determine the registered position of the ultrasound 'slice' within the 3D surgical scene. However, within the stereo display, the precise means by which the left and right operative video feeds are combined with the ultrasound texture will have a significant impact on the surgeon's ability to appreciate any observed subsurface structure.
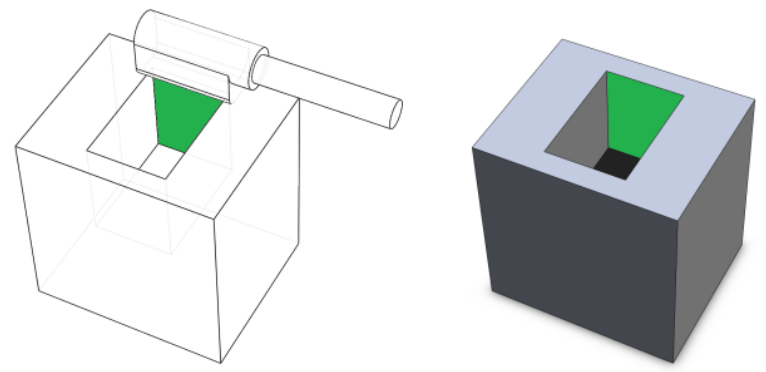

Figure 4. Geometry of windowed overlay option showing transducer and back wall (green).

Three options were made available in this study. In each case, the ultrasound image is rendered into the left and right views of the surgical scene in accordance with the stereo camera intrinsic and extrinsic parameters. The first two options comprise overwriting and alpha-blending of the ultrasound texture. The third, and perhaps most compelling option requires that a window, perpendicular to the 
ultrasound image, is removed from the original left and right images. Figure 4 illustrates the underlying geometrical arrangement with wireframe and solid views of a simple model. The window is also rendered into the alpha channel of the output buffers, such that per-pixel blending operations can be used subsequently to clip the 'vertical' quadrilaterals. The 'back wall' of the ultrasound image is rendered in this manner, together with any measurement gradations. Finally, any visible 'side walls' of the hole are added - again, with measurement gradations if enabled. Together, these features and their respective realisations in the left and right camera views help give the observer the illusion that part of the target organ's surface and interior have been cut away [14]. Employing PVA cryogel kidney phantom models, figure 5 illustrates the overlay options monoscopically.

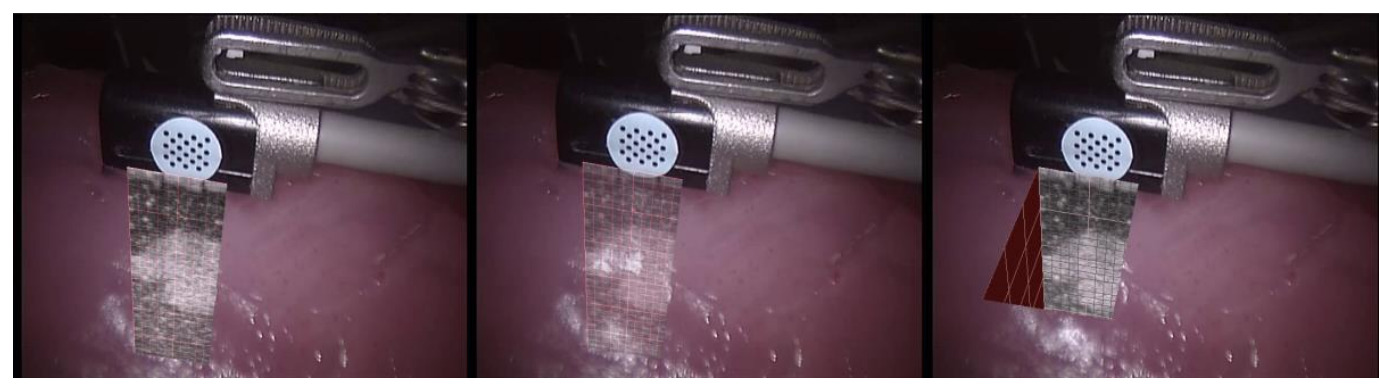

Figure 5. Ultrasound overlay schemes: overwrite (left), alpha-blend (middle) and window (right).

\section{Probe calibration}

Preliminary work [8] described a general probe calibration method applicable to the UST-533 microsurgery probe. In total, seven degrees of freedom were determined, ultimately resulting in the transformation that takes pixels in the ultrasound image to the correct position in the probe pattern coordinate frame. They comprised an image scale factor and a rigid 3D rotation and translation.

While in practice the method was capable of yielding very accurate calibrations, the relatively high dimensionality of the parameter space meant that the results were very sensitive to small deviations in the input data, e.g. the centres of the Z-phantom wire images in the reference scans. A more robust scheme was required that exploited features of the ultrasound cart and the relatively simple geometry of the transducer casing in this instance.

To this end, the pixel-to-millimetre scale factor is now determined directly using the ultrasound cart's built in measurement capability. Careful alignment of the circular dot pattern axes with respect to the rectilinear probe casing ensures 
that the rotation component of the calibration can be set to the identity. The $\mathrm{X}$ and $\mathrm{Z}$ components of the translation are determined by direct measurement of the transducer position relative to its outer casing. The remaining degree of freedom, the $\mathrm{Y}$ translation, is determined by alignment of the direct view of a single crosswire phantom feature [8] with its ultrasound image overlay.

\section{Probe decontamination and deployment}

Driven by a ProSound $\alpha-10$ cart, the ultrasound transducer used in this study was a UST-533 multi-frequency linear array microsurgery probe (Hitachi Aloka Medical Ltd., Tokyo, Japan). It is certified for sterilisation using a hydrogen peroxide plasma process, and in this instance a STERRAD 100NX unit was employed (Johnson \& Johnson, New Brunswick, NJ, USA). In order that the transducer can be picked up by da Vinci ProGrasp ${ }^{\mathrm{TM}}$ or Cadiere forceps instruments (Intuitive Surgical, Sunnyvale, CA, USA), a small friction fit probe clip was designed and 3D-printed in implant-grade cobalt-chrome-molybdenum superalloy using the direct metal laser sintering (DMLS) process (figure 6). This material is suitable for high pressure steam sterilisation in an autoclave.

To validate the sterilisation processes, the ultrasound probe (with KeyDot markers attached to both faces), probe clips and holding trays were deliberately contaminated and then subjected to their documented washing and sterilisation cycles. Swabs samples were taken at the reprocessing facility, both before and after cleaning, and were sent for independent microbiological analysis at an accredited laboratory, in accordance with the ISO 11137 standard. For each item and the control, the number of colony forming units (CFUs) was measured to be well within expected limits.
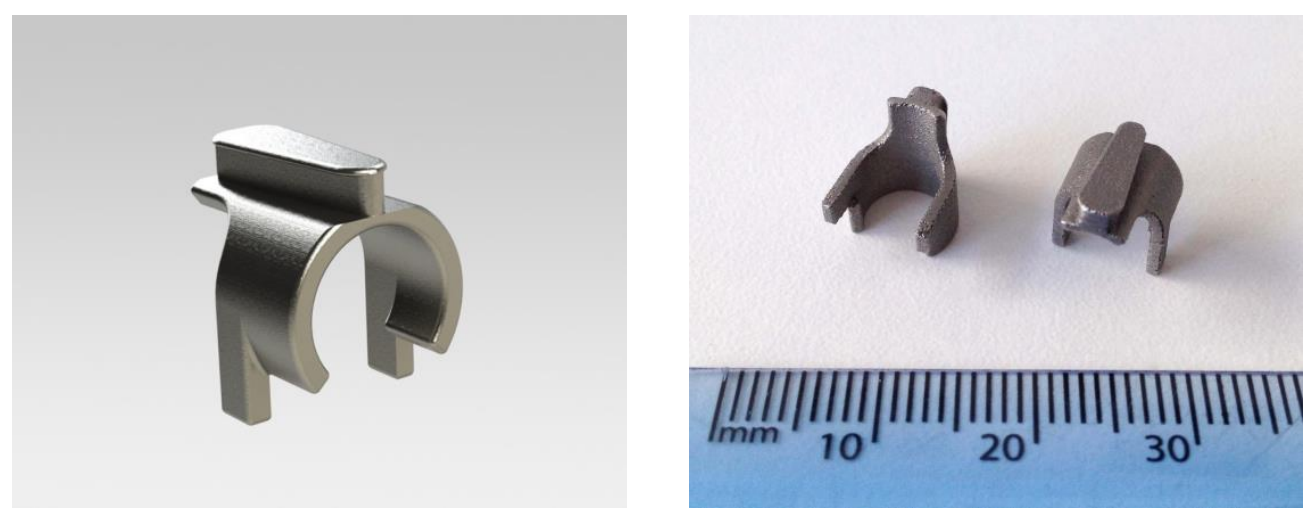

Figure 6. Ultrasound probe clip design (left) and manufactured instances with scale (right). 
Acting as an additional safety measure, a long suture is attached to the ultrasound probe clip before insertion into the patient's body through the assistant port (figure 7). In the unlikely event that the clip becomes detached from the transducer, the tether can be used to retrieve it through the port.

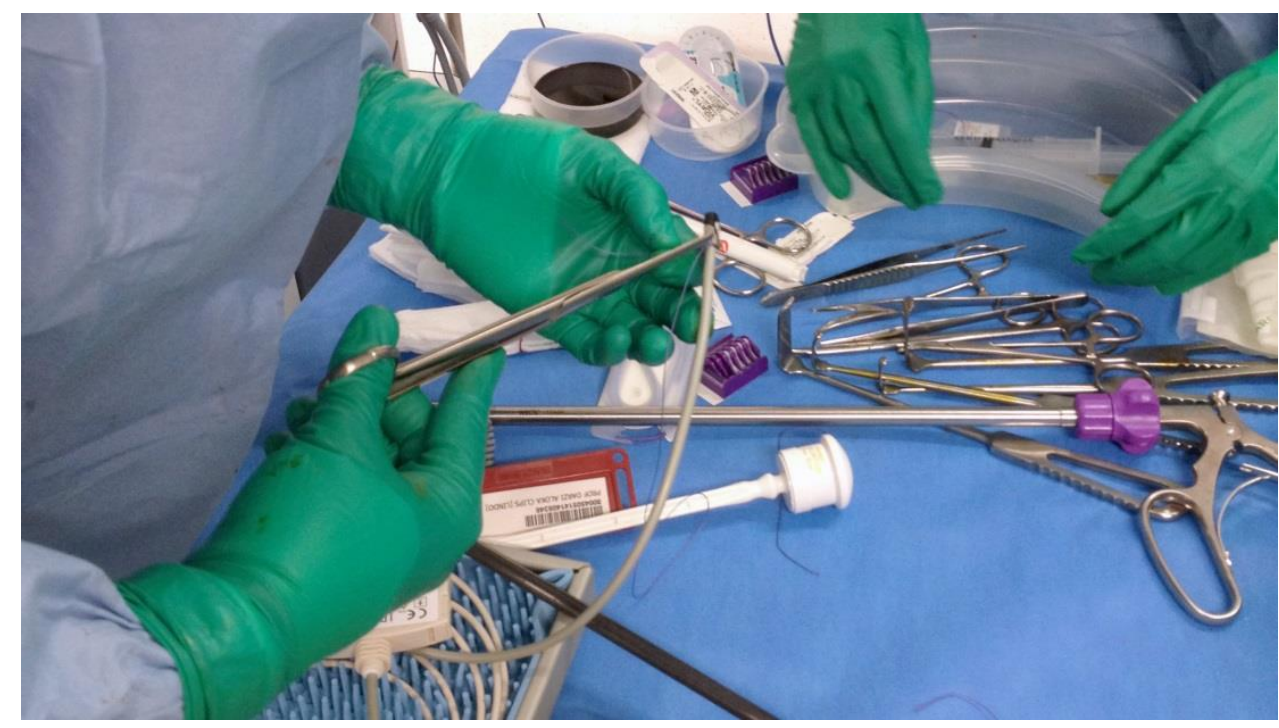

Figure 7. Tether suture being applied to ultrasound probe clip prior to insertion.

\section{Results}

\section{Probe calibration}

To assess probe calibration accuracy, eight different standard-definition camera and transducer poses were used to compare ultrasound images of the validation phantom crossing point [8] against the corresponding direct line-of-sight views, afforded when the phantom is drained of water with its door removed. The mean, standard deviation and maximum of the overlay errors were found to be $537 \mu \mathrm{m}$, $90 \mu \mathrm{m}$ and $653 \mu \mathrm{m}$, respectively, and can be seen to be an improvement over the validation results $(669 \mu \mathrm{m}, 255 \mu \mathrm{m}$ and $961 \mu \mathrm{m}$, respectively) generated by the previous 7-DOF optimisation method. For comparison, a mean stereo camera calibration reprojection error of 0.50 pixels was recorded.

\section{Operational envelope}

Physical measurements were taken to determine the operational envelope of the ultrasound probe to which the circular dot pattern marker had been attached. The 
results are summarized in table 1. The minimum and maximum attainable rotations and translations in the $\mathrm{X}, \mathrm{Y}$ and $\mathrm{Z}$ planes (each with respect to the camera's coordinate system) were recorded. Each minimum and maximum measurement was taken ten times. Rotational measurements about the $\mathrm{X}$ and $\mathrm{Y}$ axes were recorded at a distance of $55 \mathrm{~mm}$ from the endoscope tip. A camera exposure time of $1 / 85^{\text {th }}$ of a second was used. The light fountain (Karl Storz $\mathrm{GmbH}$, Tuttlingen, Germany) was set at an intensity level of $75 \%$ for the duration of the experiment. A Wolf stereo endoscope (Richard Wolf GmbH, Knittlingen, Germany) with HD camera heads was utilised in the laboratory setting.

\begin{tabular}{c|c|c|c|c|}
\multicolumn{1}{c}{} & \multicolumn{1}{c}{ range (mean) } & $\min ($ mean $)$ & $\max ($ mean $)$ & \multicolumn{1}{c}{$\max (\mathrm{std}$ dev) } \\
\cline { 2 - 5 } rotate $\mathrm{X}$ & $102.5^{\circ}$ & $-50.1^{\circ}$ & $52.4^{\circ}$ & $4.1^{\circ}$ \\
\cline { 2 - 5 } rotate $\mathrm{Y}$ & $110.3^{\circ}$ & $-52.7^{\circ}$ & $57.6^{\circ}$ & $3.4^{\circ}$ \\
\cline { 2 - 5 } translate $\mathrm{Z}$ & $73 \mathrm{~mm}$ & $13 \mathrm{~mm}$ & $86 \mathrm{~mm}$ & $4.6 \mathrm{~mm}$ \\
\cline { 2 - 5 } & &
\end{tabular}

Table 1. Operational envelope for circular dot marker pattern

Through comparison to the operational envelope results generated for the original chessboard pattern implementation [8], the extent of the incremental improvement can be calculated. An increase of $5.7 \%$ and $8.1 \%$ in the $\mathrm{X}$ and $\mathrm{Y}$ axis rotation ranges is observed, respectively. Whereas these changes are relatively modest, a substantially large increase of $74 \%$ in the $\mathrm{Z}$ translation range is also observed. This is perhaps the most significant measure of the operational envelope, as the surgeon will naturally try and orient the ultrasound probe such that the tracking pattern, and hence the registered ultrasound image itself, are perpendicular to the long axis of the endoscope.

Furthermore, qualitative observations were made by using the circular dots and chessboard pattern markers in a side-by-side configuration, such that footage could be recorded and then played back through the respective tracking algorithms. The use of the chessboard marker required more careful placement from the user with regards to its position in front of the camera. A more robust experience was found using the circular dot marker as its behaviour was less sensitive to large position changes. 


\section{Illumination levels}

Endoscopic videos of the chessboard and circular dot patterns in the same physical location were recorded, comprising a sequence of ten increasing illumination levels, equally spaced throughout the extent of the light fountain dial (figure 8), at three different distances from the endoscope tip: $15 \mathrm{~mm}$ (Near, as illustrated); 26mm (Middle); and 38mm (Far).

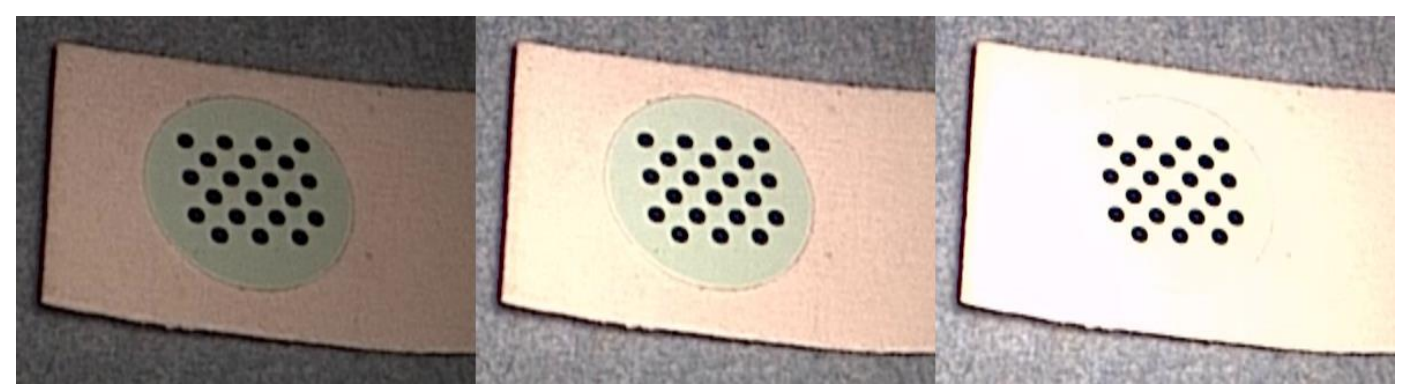

Figure 8. Range of illumination levels (reaching saturation and blooming in the right-most image).

Figure 9 illustrates that at each representative distance, the circular dots pattern performance is superior. Sensor blooming has a more deleterious effect on the sharp corners of the chessboard, in the sense that they become disconnected as pixels bleed into each other. This is in contrast to a circle, which will remain topologically identical until it disappears completely. Storing the last best tracking transformation can help overcome intermittent tracking behaviour, but only up to a point. With respect to illumination, the circular dot pattern behaves more robustly.

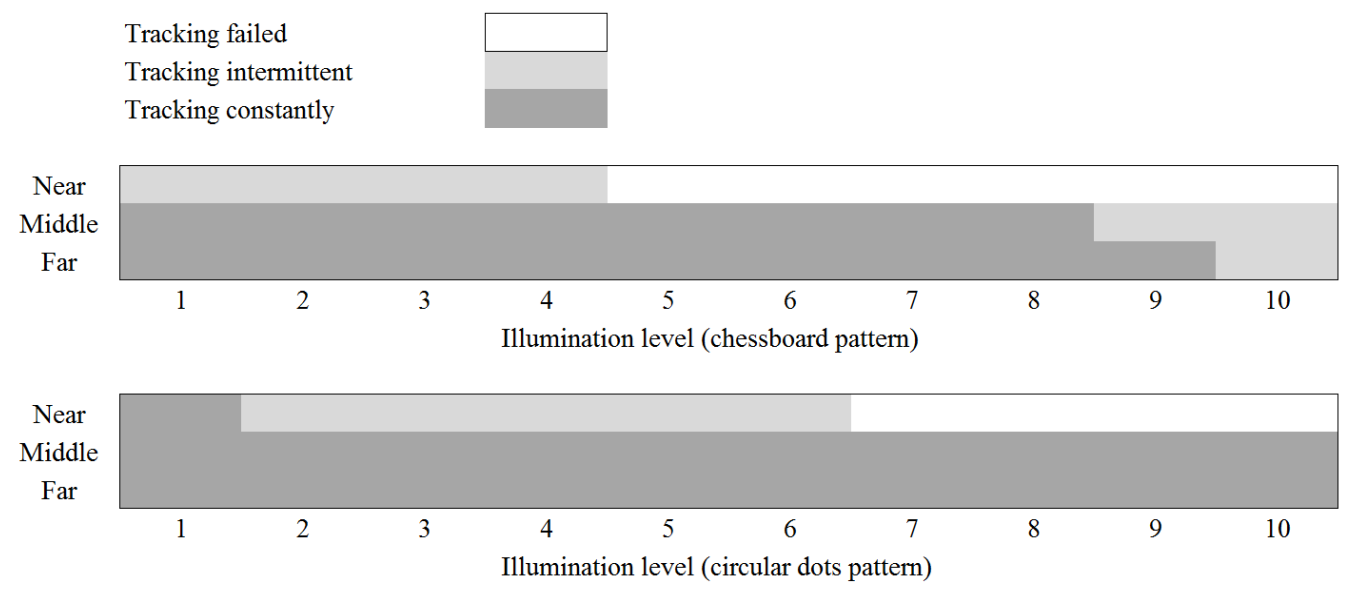

Figure 9. Tracking results over illumination range (intermittent and continuous). 


\section{Focus position}

The performance of the chessboard and circular dots pattern tracking algorithms with respect to changes in focus position (equivalently, with respect to the tracking target moving in and out of the endoscope's relatively shallow depth of field), bears a similar relation. It can be seen from figure 10 that the circular dots pattern has a wider successful tracking envelope. Closer inspection reveals that the latter algorithm is less prone to intermittent behaviour - it is therefore less susceptible to imperfections in the video input, which is inevitably noisy. Again, this can be attributed to the geometrically simpler nature of the pattern - there are no sharp corners to erode.

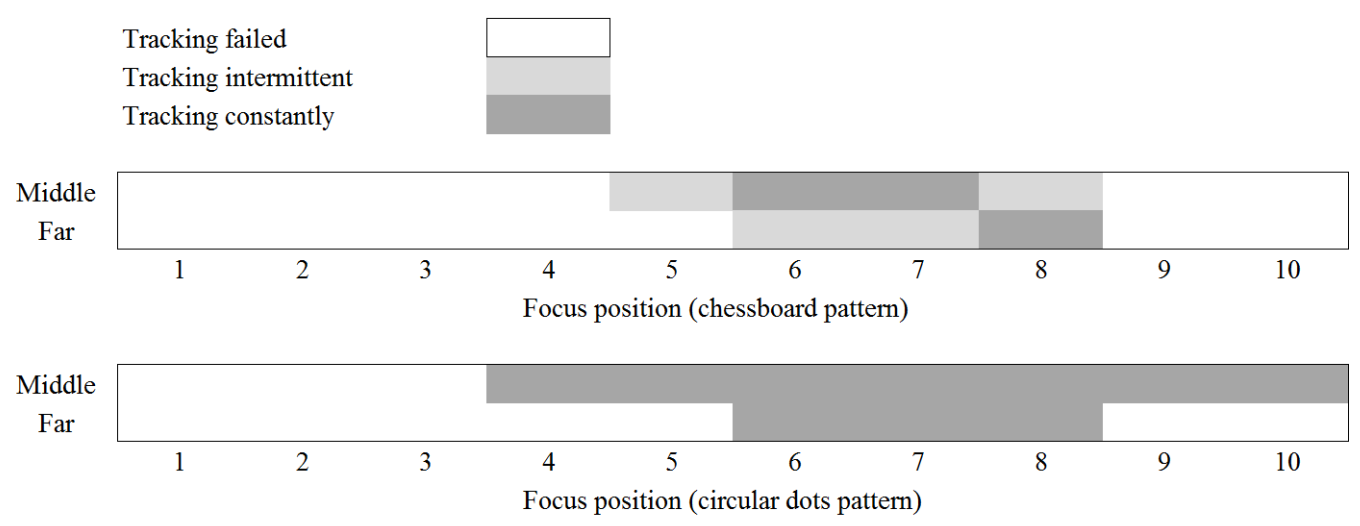

Figure 10. Tracking results over focus position range (intermittent and continuous).

Typically, during the course of an intervention, there will be periods when even a high level of robustness with respect to the target focus quality will be insufficient to accommodate the necessarily changing spatial relationship between camera and surgical scene. It is therefore imperative that any practical system has both robustness and a mechanism for rapid recalibration when the extent of the scene change is too great. It is worth noting that the relationship between the ultrasound image and probe pattern coordinate system remains constant, and therefore it is necessary only to repeat the endoscope intrinsic parameter calibration. This can be achieved using the tracking pattern itself, in conjunction with a pre-calibrated model of endoscope focus characteristics across the entire focus range [15].

\section{Tracking performance}

Minimal video processing delay is critical to the successful uptake and deployment of any image guidance system in clinical practice. Using different 
camera systems with different resolutions, and measuring tracking processing time on the live system (where the implementation is instrumented with highresolution timers), table 2 compares the performance of the original chessboard pattern versus the new circular dot pattern. In the low resolution case (PAL), typical of many laboratory configurations, the blob detector algorithm with predictive cropping significantly outperforms the original chessboard approach. It should be noted that in this instance, unlike the HD case, the initial corner detector was not accelerated with CUDA [8] since without it, acceptable frame rates and delays are achievable at the lower resolution.

However, most systems currently in clinical practice will be using HD resolution, and so the more important set of results are to be found in the lower two rows. While the performance of the two algorithms is very similar when the tracking target is at the position closest to the endoscope, the predictive cropping comes into play significantly when the target is positioned at a distance (processing times highlighted in yellow $-11 \mathrm{~ms}$ versus $17 \mathrm{~ms}$ ). This is due to the fact that the size of the cropping window is proportional to the observed spacing between the circular dots. The further away the pattern, the smaller the image area that must undergo thresholding, contour delineation and blob detection.

\begin{tabular}{|cc|cccc|}
\multicolumn{1}{c}{ resolution } & pattern & far & near & empty & failure \\
\hline PAL & circular dots & $5 \mathrm{~ms}$ & $12 \mathrm{~ms}$ & $13 \mathrm{~ms}$ & $14 \mathrm{~ms}$ \\
$720 \times 576$ & chessboard & $29 \mathrm{~ms}$ & $29 \mathrm{~ms}$ & $25 \mathrm{~ms}$ & $30 \mathrm{~ms}$ \\
\hline HD & circular dots & $11 \mathrm{~ms}$ & $22 \mathrm{~ms}$ & $75 \mathrm{~ms}$ & $60 \mathrm{~ms}$ \\
$1920 \times 1080$ & chessboard & $17 \mathrm{~ms}$ & $21 \mathrm{~ms}$ & $11 \mathrm{~ms}$ & $22 \mathrm{~ms}$ \\
\hline
\end{tabular}

Table 2. Tracking performance comparison at different resolutions and distances

The relatively fast processing time of $11 \mathrm{~ms}$ in the HD / chessboard case, where the image was empty (i.e. devoid of any pattern), can be attributed to the fact that the prevailing algorithm has an early exit clause which is invoked when the number of detected corners falls below a predefined threshold. Moreover, the high processing times of $75 \mathrm{~ms}$ and $60 \mathrm{~ms}$, for the HD circular dots case, represent the delay that could be expected if predictive cropping were not enabled. Once tracking has been enabled, the processing will run at this speed until the algorithm 
'locks on' to the dots pattern. This initial period is typically very brief, and subjectively does not present any issues.

\section{Human case report}

The following report documents the first human use of circular dot patterns for tracking in ultrasound-guided robotic partial nephrectomy. The patient recruited to this study was a 67 year old female with a tumour approximately $3.5 \mathrm{~cm}$ in diameter presenting in the left kidney. A preoperative CT scan of the urinary tract, with contrast, at high resolution, was acquired approximately two months prior to the date of the intervention, using a Brilliance 64-channel scanner (Philips Healthcare, Amsterdam, The Netherlands). The axial slice thickness was $2 \mathrm{~mm}$, with an in-plane resolution of 512 by 512 voxels.

Informed and written consent was obtained from the patient prior to the intervention under the ethical protocol for the study, entitled "Improving Outcomes in Robotic and Endoscopic Surgery using Augmented Reality Guidance". The most recent substantial amendment to this study (REC reference 07/Q0703/24) was given a favourable ethical opinion by the NRES Committee London - Dulwich. The da Vinci stereo HD $30^{\circ}$ endoscope was calibrated [13] at the outset of the procedure during the robot preparation phase. The mean reprojection error was recorded as 0.46 pixels.

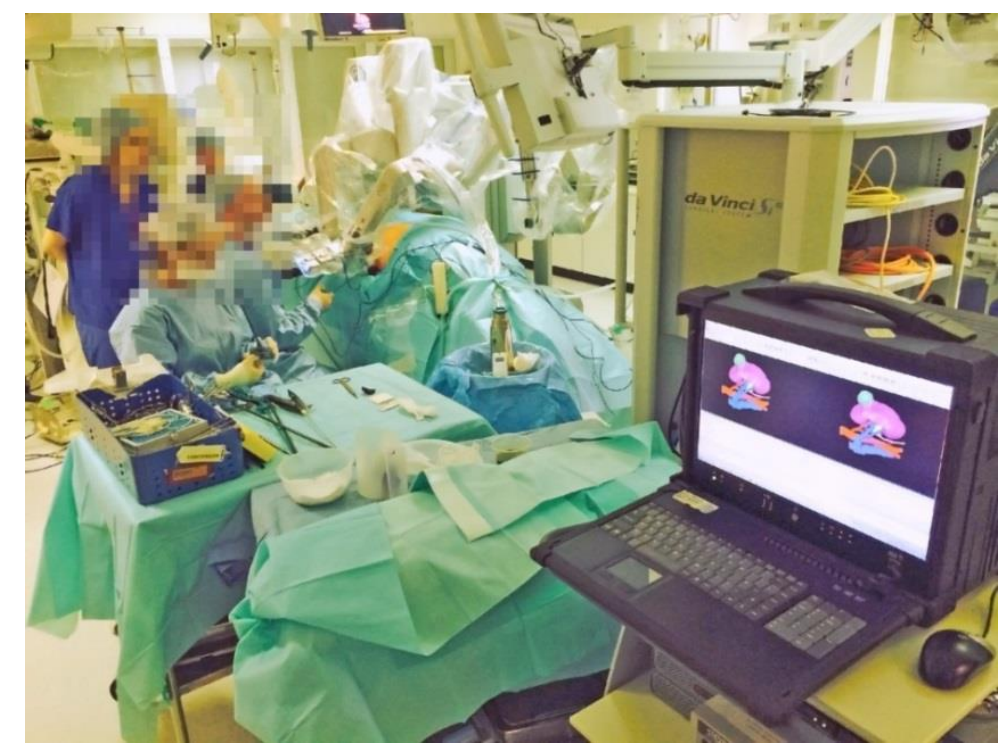

Figure 11. Theatre layout showing the image guidance portable workstation in the foreground. 
The preoperative imaging was segmented using ITK-SNAP [16], and mesh-based renderings of the left kidney, tumour, vasculature and ureter were provided in the surgical console in anticipation of the intraoperative ultrasound probe deployment (figures 11 and 12). The structure of the left renal hilar vasculature was relatively uncomplicated (single branch from the aorta, one proximal bifurcation and another distal bifurcation), which permitted the application of a single arterial clamp proximal to the aorta in order to achieve a period of warm ischemia, during which the resection took place.

Although not directly pertinent to this study, these details are included in the case report to put the developmental process in a clear clinical context, and to emphasise the staged approach where different imaging modalities are employed at different stages of the intervention. An integrated navigation platform such as this, offering multimodal, multiscale guidance can give the surgeon the most appropriate information (spatial, topological or otherwise) at the key junctures, to ensure the best patient outcomes.

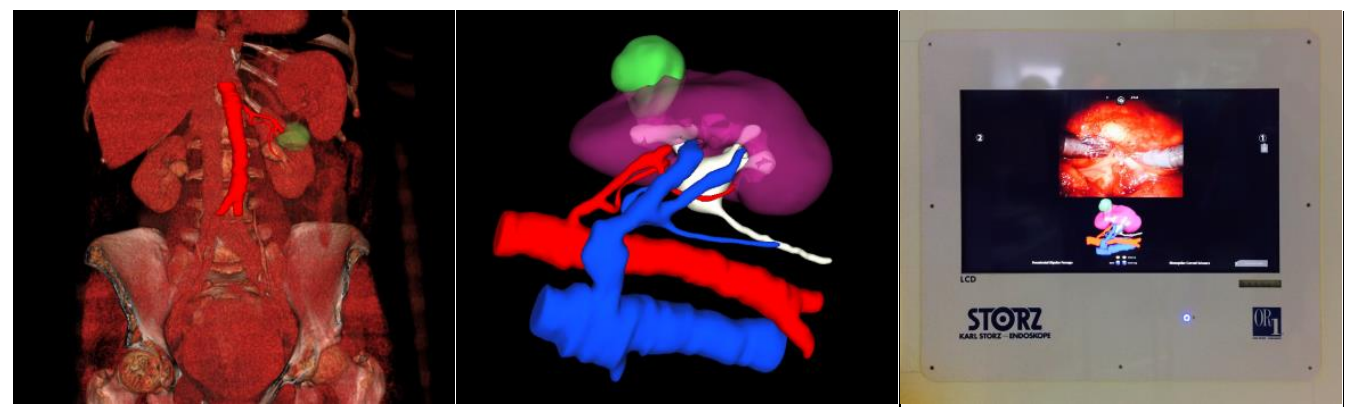

Figure 12. Console and assistant views of left kidney showing vasculature and tumour location.

Immediately prior to resection, the registered ultrasound overlay was used to confirm the location of the tumour. As can be seen in figure 13, the registered scan clearly demonstrates the interface between the normal parenchyma and tumour. The light red gradations on the image denote intervals of $1 \mathrm{~mm}$. Anecdotal surgeon feedback immediately following the case suggested that, while the accurately registered overlay capability was efficacious, the partial occlusion or blending of the surgical scene was a source of distraction from time to time. Negative tumour margins were reported postoperatively. 


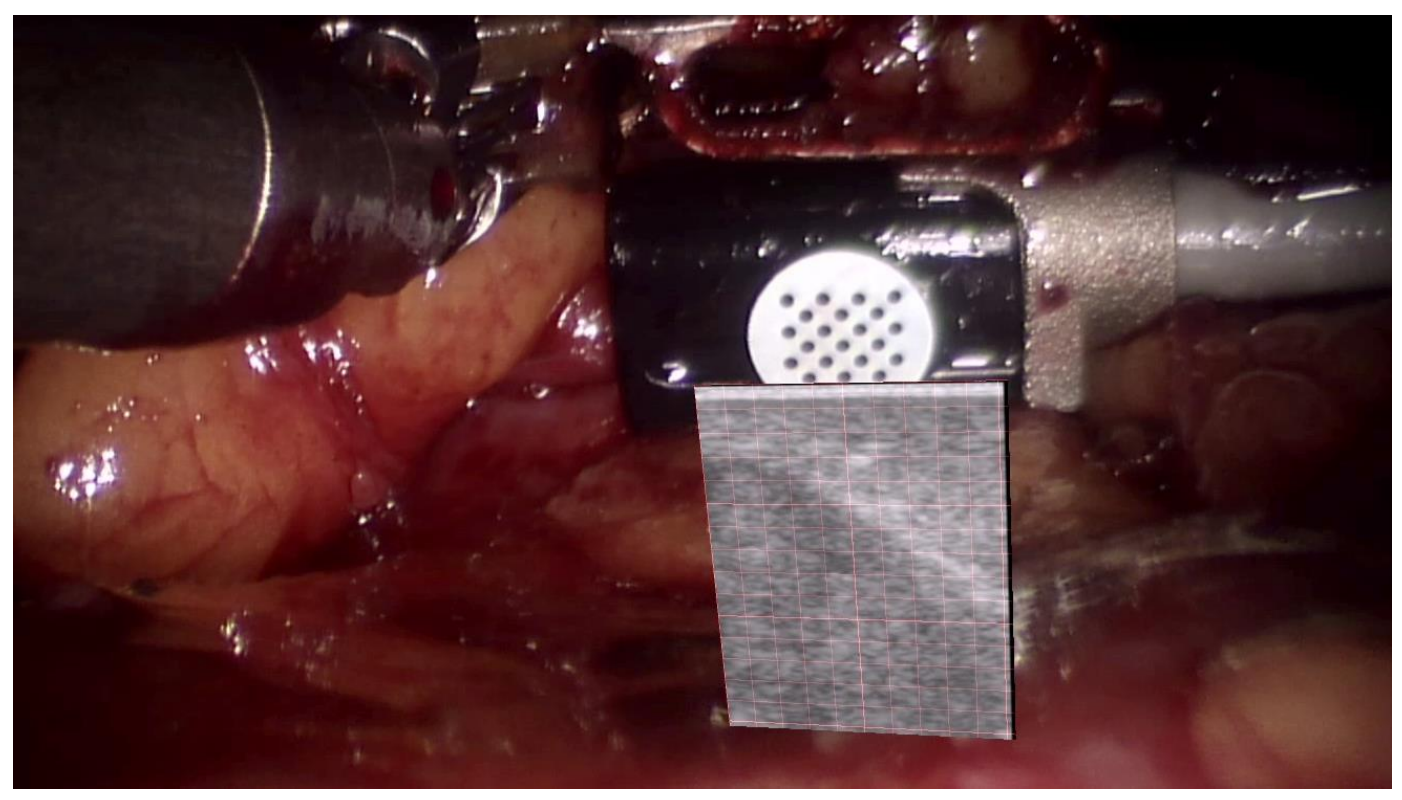

Figure 13. Still frame from left console feed illustrating registered image of tumour interface.

\section{Conclusion}

Both quantitative and qualitative results indicate that circular dot patterns are a significant improvement over the previous chessboard implementation, and a successful human trial heralds more extensive clinical evaluation. The observed level of robustness aids the development of 3D volume reconstructions based on freehand slice acquisition, which may help overcome some of the psychophysical barriers to interpretation. Paradoxically, the action of ultrasound scanning and overlay, while improving the surgeon's ability to appreciate the tumour location, impairs their ability to perform resection at the same time. Therefore, important research remains to be undertaken in this area.

One approach that has received some attention involves placing the ultrasound transducer in a location behind or alongside the organ of interest, where it is also invisible or only partially visible through the endoscope, but at a distance from the dissection site. This requires an unavoidable intraoperative registration procedure, for example, using a tracked tool to generate feature correspondences at the airtissue boundary [17], or using a similar method employing the photoacoustic effect [18] where sound waves are generated following light absorption at visible points. Thus far, no solution represents a panacea; in this instance, the registration is dependent on the prevailing camera position.

While the core of the tracking algorithm described herein uses an open source implementation, thereby promoting repeatability, calibration and tracking 
methods need to be optimised for the range of dedicated and commercially available robotic ultrasound probes now on the market. Furthermore, while the robot kinematics alone are insufficiently accurate to register the endoscopic and ultrasound views over an extended time period, a complementary scheme could mitigate those situations where tracking failures inevitably arise.

\section{Acknowledgements}

The authors are grateful for support from The Hamlyn Centre and the NIHR Biomedical Research Centre funding scheme, and would like to thank members of the operating theatre team at the Surgical Innovation Centre, St Mary's Hospital, Paddington, London.

\section{Compliance with Ethical Standards}

Conflict of Interest: The authors declare that they have no conflict of interest.

Ethical approval: All procedures performed in studies involving human participants were in accordance with the ethical standards of the institutional and/or national research committee and with the 1964 Helsinki declaration and its later amendments or comparable ethical standards. This article does not contain any studies with animals performed by any of the authors.

Informed consent: Informed consent was obtained from all individual participants included in the study.

\section{References}

[1] Hughes-Hallett A, Mayer E, Marcus H, Cundy T, Pratt P, Darzi A, Vale J (2013) Augmented reality partial nephrectomy: examining the current status and future perspectives. Urology, 83(2). Elsevier, 266-273

[2] Hughes-Hallett A, Pratt P, Mayer E, Martin S, Darzi A, Vale J (2014) Image guidance for all TilePro display of 3-dimensionally reconstructed images in robotic partial nephrectomy. Urology, volume 84(1). Elsevier, 237-243

[3] Sun M, Wagner A, San Francisco I, Brook A, Kavoussi L, Russo P, Steele G, Viterbo R, Pedrosa I (2012) Need for intraoperative ultrasound and surgical recommendation for partial nephrectomy: correlation with tumor imaging features and urologist practice patterns. Ultrasound Q, 28 (1) 21-27

[4] Leven J, Burschka D, Kumar R, Zhang G, Blumenkranz S, Xiangtian D, Awad M, Hager G, Marohn M, Choti M, Hasser C, Taylor R (2005) DaVinci Canvas: A Telerobotic Surgical System with Integrated, Robot-Assisted, Laparoscopic Ultrasound Capability. MICCAI, LNCS 3749. Springer, Berlin, 811-818 
[5] Schneider C, Dachs, G, Hasser C, Choti M, DiMaio S, Taylor R (2010) Robot-Assisted Laparoscopic Ultrasound. Information Processing and in Computer-Assisted Interventions, LNCS 6135. Springer, Berlin, 67-80

[6] Schneider C, Guerrero J, Nguan CY, Rohling R, Salcudean SE (2011) Intra-operative "PickUp" ultrasound for robot assisted surgery with vessel extraction and registration: a feasibility study. In International conference on information processing in computer assisted interventions. Springer, Berlin, 122-132

[7] Cheung CL, Wedlake C, Moore J, Pautler SE, Peters TM (2010) Fused video and ultrasound images for minimally invasive partial nephrectomy: a phantom study. MICCAI, Part III, LNCS 6363. Springer, Berlin, 408-415

[8] Pratt P, Di Marco A, Payne C, Darzi A, Yang G-Z (2012) Intraoperative ultrasound guidance for transanal endoscopic microsurgery. MICCAI, Part I, LNCS 7510. Springer, Berlin, 463-470

[9] Jayarathne U, McLeod AJ, Peters T, Chen E (2013) Robust intraoperative US probe tracking using a monocular endoscopic camera. MICCAI, Part III, LNCS 8151. Springer, Berlin, 363-370

[10] Hughes-Hallett A, Pratt P, Mayer E, Di Marco A, Yang G-Z, Vale J, Darzi A (2013) Intraoperative ultrasound overlay in robot-assisted partial nephrectomy: first clinical experience. European Urology, 65 (3). Elsevier, 671-672

[11] Bradski G, Kaehler A (2008) Learning OpenCV: Computer vision with the OpenCV library. O'Reilly Media, Inc.

[12] Suzuki S, Abe K (1985) Topological structural analysis of digitized binary images by border following. Computer vision, graphics, and image processing 30 (1), 32-46

[13] Zhang Z (2000) A flexible new technique for camera calibration. IEEE Transactions on Pattern Analysis and Machine Intelligence 22(11), 1330-1334

[14] Fuchs H, State A, Pisano E, Garrett W, Hirota G, Livingston M, Whitton M, Pizer S (1996) Towards performing ultrasound-guided needle biopsies from within a head-mounted display. Visualization in Biomedical Computing, LNCS 1131, Springer, 591-600

[15] Pratt P, Bergeles C, Darzi A, Yang G-Z (2014) Practical intraoperative stereo camera calibration. Proceedings of the international conference on medical image computing and computer-assisted intervention. Part II, LNCS 8674. Springer, Berlin, 667-675

[16] Yushkevich P, Piven J, Cody-Hazlett H, Gimpel-Smith R, Ho S, Gee J, Gerig G (2006) Userguided 3D active contour segmentation of anatomical structures. Neuroimage 31(3), 1116-1128

[17] Yip M, Adebar T, Rohling R, Salcudean S, Nguan C (2010) 3D Ultrasound to Stereoscopic Camera Registration through an Air-Tissue Boundary. MICCAI, LNCS 6362. Springer, Berlin, 626-634

[18] Cheng A, Kang J, Taylor R, Boctor E (2012) Direct 3D Ultrasound to Video Registration Using Photoacoustic Effect. MICCAI, LNCS 7511. Springer, Berlin, 552-559 